\title{
2D photos are great, but what about 3D imaging?
}

\author{
Senmao Li ${ }^{1}$ Alexander C. Rokohl ${ }^{1} \cdot$ Yongwei Guo $^{2} \cdot$ Ludwig M. Heindl $^{1}$ (1)
}

Received: 3 October 2021 / Revised: 3 October 2021 / Accepted: 24 October 2021 / Published online: 30 October 2021

(c) The Author(s) 2021

Dear Editor,

With great interest, we read the article "Analysis of surgical outcome after upper eyelid surgery by computer vision algorithm using face and facial landmark detection" by our highly appreciated colleagues İlke Bahçeci Şimşek and Can Şirolu [1].

The authors provided us some very important insights into a computer vision algorithm using the face and facial landmark detection system for normalizing and calibrating photographs. This system offers a simple, standardized, objective, and reproducible assessment method for patients who underwent upper eyelid blepharoplasty. We fully agree that this is a first step of using artificial intelligence for evaluating the outcome of blepharoplasty in a more standardized fashion. However, there are some shortcomings in this study that might be addressed.

In this study [1], the original photos were captured by a Nikon D90 SLR (Single Lens Reflex) digital camera. Therefore, all the measurements were based on two-dimensional (2D) photos. Traditionally, craniofacial anthropometry is mainly obtained by direct caliper measurements or 2D photogrammetry. However, direct anthropometry using a caliper is not only time-consuming but also extremely dependent on patient's compliance. Nowadays, informed patients are increasingly aware that these technologies cannot exactly acquire and quantify the complicated three-dimensional (3D) morphology of human faces in a standardized manner [2]. In addition, 2D photogrammetry falls short of proper 3D facial depth, and inaccuracy has been reported [2]. Three-dimensional imaging not only presents, in particular, the periocular region more vividly and realistically to both the surgeon and the patient but is also significantly more

Ludwig M. Heindl

ludwig.heindl@uk-koeln.de

1 Department of Ophthalmology, University of Cologne, Faculty of Medicine and, University Hospital of Cologne, Kerpener Strasse 62, 50937 Cologne, Germany

2 Eye Center, Second Affiliated Hospital, School of Medicine, Zhejiang University, Hangzhou, China accurate compared to $2 \mathrm{D}$ images due to the use of the $3 \mathrm{rd}$ dimension.

In recent decades, novel non-invasive 3D imaging technologies are replacing both classic direct anthropometry (using rulers and calipers) and 2D digital photogrammetry. Three-dimensional imaging technologies allow operators to assess facial or mammary morphology and their alterations over time or after treatment, as well as differences between genders, ages, or ethnicities by analyzing the highly detailed metrical measurements on 3D surfaces. According to our studies, stereophotogrammetry and our landmark location protocol (Fig. 1) for the periocular region yielded very good reliability for a series of 2D and 3D liner, curvilinear, and angular measurements [2]. Furthermore, this 3D imaging system and landmark protocol was applied on describing the periocular region morphology and assessing its relationship with aesthetics in a European population [3]. In addition, we have applied this 3D imaging system for medial canthal tendon laxity and lower eyelid tension $[4,5]$.

In summary, more precise and vivid results could be achieved by using 3D imaging with a validated landmark protocol, instead of simple 2D photos. In addition, it would be interesting to combine 3D imaging and a computer vision algorithm with a periocular landmark detection system for improving the accuracy as well as provide patients best care using state-of-the-art technologies including artificial intelligence. 
Fig. 1 Fifty-two three-dimensional anthropometric landmarks of the periocular region elucidated in a two-dimensional modality. Five prime points are first located on 3D surface models, including the endocanthion, exocanthion, pupillary center, as well as the medial and lateral corneoscleral limbus (horizontal to the pupillary center)

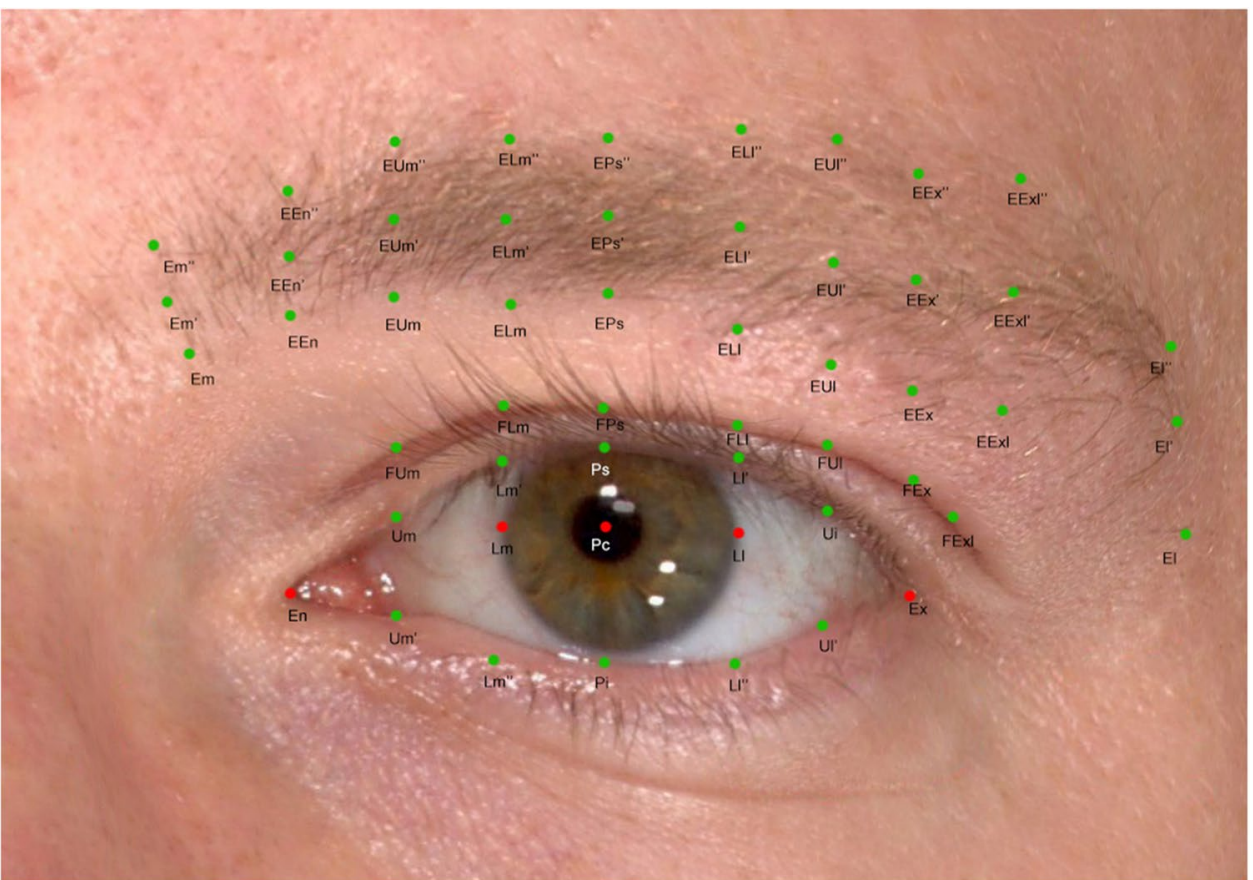

Funding Open Access funding enabled and organized by Projekt DEAL. This study was supported by the "Fundamental Research Funds for the Central Universities" (No. 2021FZZX005-15) and the Koeln Fortune Program/Faculty of Medicine, University of Cologne, Cologne, Germany (No. 2680148101).

\section{Declarations}

Ethics approval and consent All procedures performed in this study involving human participants were in accordance with the ethical standards of the institutional research committee and with the 1964 Helsinki declaration and its later amendments or comparable ethical standards. No animal subjects were included in this study.

Conflict of interest The authors declare no competing interests.

Open Access This article is licensed under a Creative Commons Attribution 4.0 International License, which permits use, sharing, adaptation, distribution and reproduction in any medium or format, as long as you give appropriate credit to the original author(s) and the source, provide a link to the Creative Commons licence, and indicate if changes were made. The images or other third party material in this article are included in the article's Creative Commons licence, unless indicated otherwise in a credit line to the material. If material is not included in the article's Creative Commons licence and your intended use is not permitted by statutory regulation or exceeds the permitted use, you will need to obtain permission directly from the copyright holder. To view a copy of this licence, visit http://creativecommons.org/licenses/by/4.0/.

\section{References}

1. Bahceci Simsek I, Sirolu C (2021) Analysis of surgical outcome after upper eyelid surgery by computer vision algorithm using face and facial landmark detection. Graefes Arch Clin Exp Ophthalmol 259(10):3119-3125. https://doi.org/10.1007/s00417-021-05219-8

2. Guo Y, Hou X, Rokohl AC, Jia R, Heindl LM (2020) Reliability of periocular anthropometry: a comparison of direct, 2-dimensional, and 3-dimensional techniques. Dermatol Surg 46(9):e23-e31. https://doi.org/10.1097/DSS.0000000000002243

3. Guo Y, Schaub F, Mor JM, Jia R, Koch KR, Heindl LM (2020) A simple standardized three-dimensional anthropometry for the periocular region in a European population. Plast Reconstr Surg 145(3):514e-523e. https://doi.org/10.1097/PRS.0000000000 006555

4. Hou X, Rokohl AC, Meinke MM, Li S, Liu J, Fan W, Lin M, Jia R, Guo Y, Heindl LM (2021) A novel standardized distraction test to evaluate lower eyelid tension using three-dimensional stereophotogrammetry. Quant Imaging Med Surg 11(8):3735-3748. https://doi.org/10.21037/qims-20-1016

5. Hou X, Rokohl AC, Meinke MM, Liu J, Li S, Fan W, Lin M, Jia R, Guo Y, Heindl LM (2021) Standardized three-dimensional lateral distraction test: its reliability to assess medial canthal tendon laxity. Aesthetic Plast Surg. https://doi.org/10.1007/ s00266-021-02440-y

Publisher's note Springer Nature remains neutral with regard to jurisdictional claims in published maps and institutional affiliations. 